\title{
Psychological pathways between type D personality and COVID-19
}

\begin{abstract}
BACKGROUND
Patients with a type D personality have worse social functioning and mental health and more affective constraints than non-type D personalities. They have a negative outlook on life and health-related issues. The aim of this study was to examine the mediating role of stress and anxiety in the relationship between type $D$ personality and COVID-19 by adjustment of the effect of demographic characteristics and perceived symptoms as confounder variables.
\end{abstract}

\section{PARTICIPANTS AND PROCEDURE}

A total of 196 patients out of those suspected of having COVID-19 and visiting the reference hospitals were selected. They had completed the type D personality and the anxiety and stress scales along with their hospital admission form before undergoing COVID-19 testing. After their COVID-19 test, the participants were divided into two groups based on their disease, an infected group $(n=90)$ and a non-infected group $(n=106)$.

\section{RESULTS}

Type D personality has no significant direct effect on infection with the disease, but taking into account the mediating variable of stress, the odds of an event in those with type D personality is 2.21 times higher than those without this personality $(p=.027)$ and, taking into account the mediating variable of anxiety, having a type $D$ personality increases the odds of an event by 2.62 times $(p=.011)$, holding demographic characteristics and perceived symptoms constant.

\section{CONCLUSIONS}

Given the indirect relationship between COVID-19 and type $\mathrm{D}$ personality, the mediating variables of stress and anxiety can be considered full mediating variables.

KEY WORDS

type D personality; COVID-19; stress; anxiety

ORGANIZATION - 1: Tabriz University of Medical Sciences, Tabriz, Iran · 2: Khoy University of Medical Sciences, Khoy, Iran AUthors' Contributions - A: Study design - B: Data collection - C: Statistical analysis · D: Data interpretation .

E: Manuscript preparation · F: Literature search · G: Funds collection

Corresponding author - Neda Gilani, Ph.D., Department of Statistics and Epidemiology, Emergency Medicine Research Team, Tabriz University of Medical Sciences, Golgasht Str., 5165665931 Tabriz, Iran, e-mail: gilanin@tbzmed.ac.ir to CITE this ARTICLE - Shahsavarinia, K., Javadivala, Z., Allahverdipour, H., Mousavi, Z., Hamidi, F., Saadati, M.,

Taghizadieh, A., Amiri, P., \& Gilani, N. (2022). Psychological pathways between type D personality and COVID-19.

Health Psychology Report, 10(1), 20-30. https://doi.org/10.5114/hpr.2021.110933 


\section{BACKGROUND}

The outbreak of the new coronavirus, known as COVID-19, in late 2019 in China, and then its rapid spread throughout the world led to significant concerns in different countries. The high rate of spread of the virus has forced countries to deal with countless numbers of infected people. Although some people recover from the disease, the rate of infection is still high. This rate of infection has occurred over a span of less than one year (Chen et al., 2020).

While prevention is an effective way of dealing with infectious viral diseases, extreme fear and stress make people more sensitive, such that many people misinterpret their physical symptoms and feel that they have been infected and therefore visit hospitals and medical centers (Dymecka et al., 2021). Visiting medical centers when there are no symptoms of infection not only increases the burden on the medical system but also increases the likelihood of infection in those visiting (Schou-Bredal et al., 2021; Wang et al., 2020). Stress and anxiety weaken the immune system and suppress its defense mechanism against viral diseases. In people who experience high levels of stress, dramatic changes can be observed in both the cellular and humoral immune systems. The changes in the humoral immune system include reductions in serum antibody titers and the rate of salivary $\operatorname{IgA}$ secretion in stressed people (Reed \& Raison, 2016).

Studies conducted in the past decade have led to major advances in understanding the effect of personality type on clinical and psychological matters (Mols \& Denollet, 2010). The 'D' in 'type D personality' signifies a distressed person, and this formulation was first proposed by Denollet et al. (1995). Type D personality is defined as a distressed personality that includes two traits concurrently, namely negative affectivity (a tendency to experience negative emotions) and social inhibition (a tendency to show inhibition in expressing oneself in social interactions) (Mols \& Denollet, 2010). In fact, it is the concurrency of negative affectivity and the inability to deal with this affectivity that determine this personality type (Kupper \& Denollet, 2007). A type D personality indicates an interaction between particular traits that may have harmful health consequences (Kupper \& Denollet, 2007).

Initially, most studies on type D personality focused on its prevalence and effects in patients with cardiovascular disorders (Raykh et al., 2021). Nonetheless, in recent years, the interest in investigating the type D personality as a risk factor in the general public instead of in cardiac patients has increased (Kim et al., 2017; Zoghipaidar et al., 2020). This type of personality has a strong and stable relationship with perceived anxiety and stress, such that it is a predictor of anxiety and stress. In other words, people with a type D personality report high levels of chronic tension and stress (Borkoles et al., 2018; Denollet \& Pe- dersen, 2009). One study showed that cancer patients with a type $\mathrm{D}$ personality are more dissatisfied with the information they are given about their disease than cancer patients not regarded as type D (You et al., 2018). A study reported that type D patients believed that their disease was more serious and longer-lasting and reported more symptoms and attributed them to their disease. Moreover, they were more concerned and more emotionally influenced by their disease.

The type D personality has a negative relationship with coherence and a positive relationship with outcomes, timetable, identity, concerns, and emotional expression (Aguayo-Carreras et al., 2020). Patients with a type D personality have worse social functioning and mental health and more affective constraints as well as less vitality than non-type D personalities. They have a negative outlook on life and health-related issues (Aguayo-Carreras et al., 2020; Mols et al., 2012). Studies refer to the role of type D personality in increasing cortisol and the activity of the immune system, while a well-known etiology hypothesis concerns immune system dysfunction, as studies have shown irregularity in the secretion of some hormones, including cortisol, and an increased secretion of IL-1, IL-6, TNF- $\alpha$, and INF-7 (Masafi et al., 2018).

Conceptually, stress and anxiety as negative affective conditions are quite distinct, but the clinical overlap between the two conditions has been approved both clinically and scientifically (Lovibond \& Lovibond, 1995). Stressful life events accelerate both anxiety disorders and chronic arousal and impaired function (Jastrowski Mano et al., 2019). Persons with type $\mathrm{D}$ personality who are high in both negative affectivity (NA) and social inhibition (SI) have high levels of stress and anxiety and are vulnerable to chronic distress and worsening health condition (Skalski et al., 2020; Tuman, 2021).

Nevertheless, the outbreak of COVID-19 and its harmful effects on society have challenged researchers from different disciplines, as the various dimensions of this disease are not very well known yet (Dymecka, 2021). Meanwhile, on the one hand, excessive anxiety and stress lead to negative outcomes, and on the other hand, the disease itself causes anxiety and stress (Allahverdipour et al., 2021; Javadivala et al., 2020; Skalski et al., 2020). The present study was therefore conducted to investigate the role of stress and anxiety and type D personality in COVID-19 in patients suspected of having this disease.

\section{PARTICIPANTS AND PROCEDURE}

\section{STUDY DESIGN AND PARTICIPANTS}

The present descriptive-analytical study was conducted after the approval of its proposal and obtaining its ethics approval from the Ethics Committee of Ta-
Type D personality and COVID-19 
briz University of Medical Sciences Institutional Review Board (consent no. R.TBZMED.REC.1398.1051) on people suspected of having COVID-19 who had presented to referral and central hospitals in Tabriz, in 2020. Based on the results obtained by Xiao et al. (2020) and taking into account $\alpha=.05$, and test power $=0.90$ and using $G^{*}$ power-3.1.2, the sample size was estimated as 30 , which was increased to 90 people given the design effect and a potential attrition bias of $30 \%$ in both groups (Xiao et al., 2020). The target population of this research consisted of all the people visiting the reference hospitals for getting a diagnosis of COVID-19 and finding out their status (infection/ non-infection) based on the eligibility criteria.

After introducing themselves and briefing the candidates on the study objectives, the voluntary nature of participation, and the confidentiality of the data, the researchers obtained informed consent from all the participants and then gave them the admission form and the study questionnaire to complete. The front page of the questionnaire contained information about the project, its significance and also the project executor's contact number to call if necessary.

Out of this population, a total of 196 people were selected for participation in the study using convenience sampling. First, they completed questionnaires while waiting for clinical examination and undergoing the COVID-19 tests. They were then assigned to groups based on their history and clinical examination and the COVID-19 tests including CBC/DIFF, CRP, LDH, spiral chest CT scan, and PCR. Those with a positive test and based on physician diagnosis were assigned to the COVID-19 (infected) group $(n=90)$, and those with a negative test and based on physician diagnosis were assigned to the non-infected group $(n=106)$.

The study inclusion criteria were: willingness to take part, minimum age of 18 years, adequate literacy for completing the data, and no neurological diseases requiring treatment by a neurologist, including no history of using antidepressants. The exclusion criteria were: unwillingness to continue participation in the study at any stage, returning incomplete questionnaires (i.e., questionnaires with $20 \%$ or more of their information completed improperly), and those with a former history of COVID-19 infection.

To comply with the hygiene protocols, each participant was given a separate pen to complete the questionnaires, and also the questionnaires received were isolated for a minimum of three weeks for prevention purposes.

\section{MEASUREMENTS}

Background data. 1) Demographic details of the patients (age, gender, marital status, education, weight, and height). 2) Disease history (history of smoking, use of alcohol, drugs, previous history of COVID-19 infection, history of underlying diseases, and history of medication use). 3) Laboratory findings (CBC/DIFF, CRP, LDH, spiral chest CT scan, PCR). 4) Symptoms (fever, cough, breath shortness, headache, body pain, nausea, vomit, diarrhea, stomach ache).

Type D Scale-16 (DS-16). The Type D Personality Scale (DS-14) was developed by Denollet (1998) as a 14-item self-reporting scale that assesses the negative affectivity (NA) and social inhibition (SI) subscales on a five-point Likert scale - 0 (true), 1 (fairly true), 2 (in between), 3 (fairly false), and 4 (false). Items $2,4,5,7,9,12$, and 13 measure $\mathrm{NA}$, and items 1 , $3,6,8,10,11$, and 14 measure SI. A cut-off point $>10$ is taken for both subscales to discern people with type D personality. Type D personality can also be taken as a continuous construct produced by multiplying its two components (Gilmour \& Williams, 2012). To assess the construct validity, the Taylor Manifest Anxiety Scale (TMAS), the extraversion and neuroticism subscales of the revised Eysenck Personality Questionnaire (EPQ), the extraversion subscale of the Minnesota Multiphasic Personality Inventory (MMPI), and the Malo-Crohn Scale were used. The TMAS and the neuroticism subscale of the EPQ were valid in measuring negative affectivity, and NA had a positive correlation with the scores of these scales (Denollet, 2000). In later studies, Denollet showed that NA and SI are highly correlated with the neuroticism and extraversion subscales of the Big Five Personality Test in healthy subjects $(R=.59-.68)$ (Denollet, 2005). Bagherian Sararoudi et al. (2011) measured Cronbach's $\alpha$ of this questionnaire and reported it as .84 for NA and .86 for SI. In the present study, Cronbach's $\alpha$ was measured as .81 for NA and .85 for SI.

Anxiety and stress scale. Lovibond's 21-item Depression, Anxiety and Stress Scale (DASS-21, short form) was used to assess anxiety and stress (Lovibond \& Lovibond, 1995). This scale measures depression, anxiety and stress separately with seven items for each. The present study assessed only anxiety (using items 2, 4, 7, 9, 15, 19, and 20) and stress (using items $1,6,8,11,12,14$, and 18) with this scale. These items are scored based on a four-point scale from 0 (did not apply to me at all) to 3 (applied to me very much or most of the time). Since DASS-21 is the short form of the original 42-item scale, the final score of each subscale must be doubled. Antony et al. (1998) performed the factor analysis of this questionnaire, and their results showed three factors, including depression, anxiety, and stress, which explained $68 \%$ of the variance of the whole scale. Henry and Crawford (2005) investigated the construct validity of the short form of DASS among 1794 adults in England. They reported a reliability coefficient of .93 for the whole scale. The validity and reliability of this scale were assessed in Iran by Samani and Joukar (2007) among 638 students of Shiraz University of Medical 
Sciences. The scale was internally consistent and the $\alpha$ coefficients reported were .87 for the stress subscale and .75 for anxiety. In the present study, the qualitative face and content validity of these scales were assessed by 11 experts and specialists in psychology and psychiatry, tool development, emergency medicine, and pulmonology subspecialty. The reliability of the questionnaire was determined using the internal consistency and Cronbach's $\alpha$ methods.

\section{STATISTICAL ANALYSIS}

Stata software (version 16) was used to analyze the data in this study. The mean and SD were reported for the quantitative data and the frequency (percentage) for the qualitative data. The missing data ranged from $0 \%$ to $0.05 \%$. To estimate the different models, we used the maximum likelihood method (ML). The normal distribution of the variables was confirmed by the Kolmogorov-Smirnov test and assessments of skewness and kurtosis $(-2<$ skewness and kurtosis $<2)$. The analysis of the mediating variables was performed using the generalized structural equation modeling (GSEM) command in Stata. Demographic characteristics and perceived symptoms were entered into each model as the confounder variables. Since the event (COVID-19) is a binary variable, the final results have been reported as the odds ratio (OR) with 95\% confidence interval. This analysis enabled us to determine the direct relationship between type $\mathrm{D}$ personality and the COVID-19 event in the absence of the mediating variables (direct effect) (path c in Figure 1). If this effect was significant, the mediators were included in the model (indirect effect). Based on this figure, stress and anxiety mediate the relationship between type D personality and COVID-19 in parallel, holding demographic characteristics and perceived symptoms constant. In other words, according to the purpose of the study, only the effects of the variables in Figure 1 are reported, but in order to obtain more accurate results, in fact, the effect of demographic characteristics and perceived symptoms are included in the model as confounder variables. The total effect is the combination of the direct and indirect effects of the type D personality variable on COVID-19. Therefore, this model had multiple mediators (stress and anxiety). We analyzed model B, which included these relevant mediators, at the same time. In model $\mathrm{B}$, the mediators would be deemed full mediating variables if the indirect effects were significant and the direct effect was non-significant. Furthermore, covariance between the error terms of stress and anxiety were determined. The strength of the mediation was computed via the variance accounted for (VAF), which is variance accounted for (explained) in the COVID-19 event by the indirect relationship (Hair et al., 2017; Beaujean \& Parker, 2020).

\section{RESULTS}

Table 1 presents participants' demographic details. The mean age of the participants was $43.35 \pm 17.63$ years. No significant difference was found between the infected and non-infected groups in terms of age, gender, education, marital status, and BMI, but there were significant differences between them in terms of urban vs. rural residence $(p=.048)$ and occupation $(p=.015)$.

\section{Figure 1}

Non-mediated (A) and mediated (B) pathways between type D personality and the occurrence of COVID-19. Path c' represents the direct effect of type D personality on COVID-19 with the mediator included in the model. The indirect effect is the product of multiplying path a by path $b$. All the models were controlled for the demographic characteristics

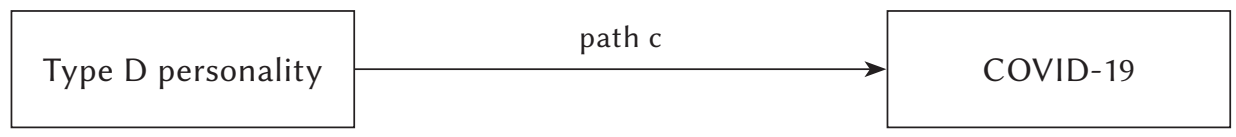

(B)

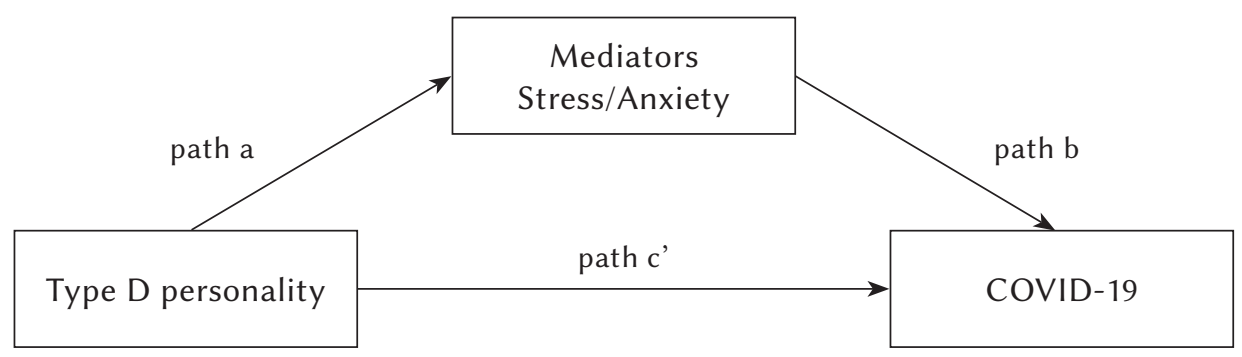


Table 1

Differences between the two groups of participants based on their demographic characteristics $(n=196)$

\begin{tabular}{|c|c|c|c|c|c|}
\hline Variables & Category & Total N (\%) & $\begin{array}{l}\text { Infected group } \\
\quad(n=90)\end{array}$ & $\begin{array}{c}\text { Non-infected } \\
\text { group }(n=106)\end{array}$ & $p^{*}$ \\
\hline \multirow[t]{4}{*}{ Age } & $\leq 30$ & $61(31.1)$ & $31(34.4)$ & $30(28.3)$ & .289 \\
\hline & $31-60$ & $99(50.5)$ & $40(44.4)$ & $59(55.7)$ & \\
\hline & $\geq 61$ & $36(18.4)$ & $19(21.1)$ & $17(16.0)$ & \\
\hline & $M \pm S D$ (years) & $43.35 \pm 17.63$ & $43.49 \pm 18.40$ & $43.23 \pm 17.04$ & \\
\hline \multirow[t]{2}{*}{ Gender } & Male & $111(56.6)$ & $51(56.7)$ & $60(56.7)$ & .993 \\
\hline & Female & $85(43.4)$ & $39(43.3)$ & $39(43.3)$ & \\
\hline \multirow[t]{5}{*}{ Education } & Middle school & $33(16.8)$ & $20(22.2)$ & $13(12.3)$ & .105 \\
\hline & High school diploma & $30(15.3)$ & $9(10.0)$ & $21(19.8)$ & \\
\hline & Associate degree & $60(30.6)$ & $31(34.4)$ & $29(27.4)$ & \\
\hline & BS (bachelor's degree) & $58(29.6)$ & $23(25.6)$ & $35(33.0)$ & \\
\hline & MS (master's degree) & $15(7.7)$ & $7(7.8)$ & $8(7.5)$ & \\
\hline \multirow[t]{2}{*}{ Marital status } & Married & $150(76.5)$ & $71(78.9)$ & $79(74.5)$ & .473 \\
\hline & Single & $46(23.5)$ & $19(21.1)$ & $27(25.5)$ & \\
\hline \multirow[t]{2}{*}{ Place of residence } & Urban & $176(89.8)$ & $85(94.4)$ & $91(85.8)$ & .048 \\
\hline & Rural & $20(10.2)$ & $5(5.6)$ & $15(14.2)$ & \\
\hline \multirow[t]{5}{*}{ Occupation } & Housewife/retired & $71(36.2)$ & $36(40.0)$ & $35(33.0)$ & .015 \\
\hline & Self-employed & $54(27.6)$ & $22(24.4)$ & $32(30.2)$ & \\
\hline & Worker (labor jobs) & $11(5.6)$ & $4(4.4)$ & $7(6.6)$ & \\
\hline & Health/medical staff & $41(20.9)$ & $25(27.8)$ & $16(15.1)$ & \\
\hline & Employee (office jobs) & $19(9.7)$ & $3(3.3)$ & $16(15.1)$ & \\
\hline \multirow[t]{5}{*}{ BMI } & Underweight & $1(0.5)$ & & $1(1.0)$ & .619 \\
\hline & Normal & $60(31.4)$ & $25(27.8)$ & $35(34)$ & \\
\hline & Overweight & $97(50.8)$ & $48(54.5)$ & $49(47.6)$ & \\
\hline & Obese & $33(17.3)$ & $15(17) 1$ & $18(17.5)$ & \\
\hline & $M \pm S D\left(\mathrm{~kg} / \mathrm{m}^{2}\right)$ & $29.14 \pm 24.64$ & $27.24 \pm 3.97$ & $30.76 \pm 33.29$ & \\
\hline \multirow[t]{3}{*}{ Type D } & Yes & $141(71.9)$ & $76(84.4)$ & $65(61.3)$ & $<.001$ \\
\hline & No & $55(28.1)$ & $14(15.6)$ & $41(38.4)$ & \\
\hline & $M \pm S D$ & $290.98 \pm 196.89$ & $362.85 \pm 203.08$ & $229.97 \pm 169.4$ & \\
\hline \multirow[t]{6}{*}{ Stress } & Normal & $94(48.0)$ & $27(30.0)$ & $67(63.2)$ & $<.001$ \\
\hline & Mild & $34(17.3)$ & $10(11.1)$ & $24(22.6)$ & \\
\hline & Moderate & $23(11.7)$ & $14(15.6)$ & $9(8.5)$ & \\
\hline & Severe & $23(11.7)$ & $18(20.0)$ & $5(4.7)$ & \\
\hline & Extremely severe & $22(11.2)$ & $21(23.3)$ & $1(0.9)$ & \\
\hline & $M \pm S D$ & $16.59 \pm 12.07$ & $22.99 \pm 12.22$ & $11.15 \pm 8.81$ & \\
\hline \multirow[t]{6}{*}{ Anxiety } & Normal & $47(24.0)$ & $6(6.7)$ & $41(38.7)$ & $<.001$ \\
\hline & Mild & $29(14.8)$ & $9(10.0)$ & $20(18.9)$ & \\
\hline & Moderate & $41(20.9)$ & $16(17.8)$ & $25(23.6)$ & \\
\hline & Severe & $39(19.9)$ & $25(27.8)$ & $14(13.2)$ & \\
\hline & Extremely severe & $40(20.4)$ & $34(37.8)$ & $6(5.7)$ & \\
\hline & $M \pm S D$ & $13.38 \pm 8.71$ & $18.27 \pm 8.86$ & $9.23 \pm 6.03$ & \\
\hline
\end{tabular}

Note. ${ }^{*}$ chi-square test. 
According to Table 2, the most common symptoms were vomiting $(83.33 \%)$, stomach ache $(77.78 \%)$, and fever $(70.49 \%)$. Also, chi-square test results showed that fever, cough, vomiting, and stomach ache were associated with a positive test outcome $(p<.05)$.

The results presented in Table 3 show a positive relationship between all the variables including NA, SI, type D, stress, and anxiety $(p<.01)$.
Figure 2 shows two models; the first model disregards the non-mediating variables (A) and the second model takes account of the mediating variables of stress and anxiety (B). In both models, the effect of demographic characteristics and perceived symptoms are considered as confounder variables. Accordingly, without taking into account the mediating variables of stress and anxiety, having a type D

\section{Table 2}

Type D personality and COVID-19

Differences between the two groups of participants based on their clinical characteristics and disease background $(n=196)$

\begin{tabular}{|c|c|c|c|c|c|}
\hline Variables & Category & Total N (\%) & $\begin{array}{l}\text { Infected group } \\
\quad(n=90)\end{array}$ & $\begin{array}{c}\text { Non-infected } \\
\text { group }(n=106)\end{array}$ & $p^{*}$ \\
\hline \multirow[t]{2}{*}{ Fever } & Yes & $61(31.12)$ & $43(70.49)$ & $18(29.51)$ & $<.001$ \\
\hline & No & $135(68.88)$ & $47(34.81)$ & $88(65.19)$ & \\
\hline \multirow[t]{2}{*}{ Cough } & Yes & $111(56.63)$ & $32(37.65)$ & $53(62.35)$ & .042 \\
\hline & No & $85(43.37)$ & $58(52.25)$ & $53(47.75)$ & \\
\hline \multirow[t]{2}{*}{ Shortness of breath } & Yes & $114(56.16)$ & $58(50.88)$ & $56(49.19)$ & .100 \\
\hline & No & $82(41.84)$ & $32(39.02)$ & $50(60.98)$ & \\
\hline \multirow[t]{2}{*}{ Headache } & Yes & $45(22.96)$ & $24(53.33)$ & $21(46.67)$ & .255 \\
\hline & No & $151(77.04)$ & $66(43.71)$ & $85(56.29)$ & \\
\hline \multirow[t]{2}{*}{ Body pain } & Yes & $93(47.45)$ & $44(47.31)$ & $49(52.69)$ & .710 \\
\hline & No & $103(52.55)$ & $46(44.66)$ & $57(55.34)$ & \\
\hline \multirow[t]{2}{*}{ Nausea } & Yes & $49(25.00)$ & $20(40.82)$ & $29(59.18)$ & .408 \\
\hline & No & $147(75.00)$ & $70(47.62)$ & $77(52.38)$ & \\
\hline \multirow[t]{2}{*}{ Vomiting } & Yes & $18(9.18)$ & $15(83.33)$ & $3(16.67)$ & .001 \\
\hline & No & $178(90.82)$ & $75(42.13)$ & $103(57.87)$ & \\
\hline \multirow[t]{2}{*}{ Diarrhea } & Yes & $31(15.82)$ & $16(51.61)$ & $15(48.39)$ & .488 \\
\hline & No & $31(15.82)$ & $74(44.85)$ & $91(55.15)$ & \\
\hline \multirow[t]{2}{*}{ Stomach ache } & Yes & $36(18.37)$ & $28(77.78)$ & $8(22.22)$ & $<.001$ \\
\hline & No & $160(81.63)$ & $62(38.75)$ & $98(61.25)$ & \\
\hline \multirow[t]{2}{*}{ Respiratory background } & Yes & $12(6.12)$ & $6(50.00)$ & $6(50.00)$ & .770 \\
\hline & No & $184(93.88)$ & $84(45.65)$ & $100(54.35)$ & \\
\hline \multirow{2}{*}{$\begin{array}{l}\text { Cardiovascular } \\
\text { background }\end{array}$} & Yes & $22(11.22)$ & $15(68.18)$ & $7(31.82)$ & .026 \\
\hline & No & $174(88.78)$ & $75(43.10)$ & $99(56.90)$ & \\
\hline \multirow[t]{2}{*}{ Digestion background } & Yes & $4(2.04)$ & $3(75.00)$ & $1(25.00)$ & .238 \\
\hline & No & $192(97.96)$ & $87(45.31)$ & $105(54.69)$ & \\
\hline \multirow[t]{2}{*}{ Neurology background } & Yes & $7(3.57)$ & $5(71.43)$ & $2(28.57)$ & .168 \\
\hline & No & $189(96.43)$ & $85(44.97)$ & $104(55.03)$ & \\
\hline \multirow{2}{*}{$\begin{array}{l}\text { Weak immunity } \\
\text { background }\end{array}$} & Yes & $7(3.57)$ & $2(28.57)$ & $5(71.43)$ & .348 \\
\hline & No & $189(96.43)$ & $88(46.56)$ & $101(53.44)$ & \\
\hline
\end{tabular}

Note. ${ }^{*}$ chi-square test. 
Table 3

Correlations and descriptive statistics for all the main variables

\begin{tabular}{lccccc}
\hline Variables & 1 & 2 & 3 & 4 & 5 \\
\hline 1. NA & - & $.74^{*}$ & $.93^{*}$ & $.24^{*}$ & $.25^{*}$ \\
2. SI & & - & $.89^{*}$ & $.30^{*}$ & $.29^{*}$ \\
3. Type D & & - & $.27^{*}$ & $.28^{*}$ \\
4. Stress & & & - & $.69^{*}$ \\
5. Anxiety & & & & - \\
M SD & $16.80 \pm 7.59$ & $15.56 \pm 5.28$ & $290.93 \pm 190.89$ & $16.58 \pm 10.01$ & $13.38 \pm 8.71$ \\
Min-Max & $0-28$ & $1-28$ & $0-784$ & $0-42$ & $0-40$ \\
\hline
\end{tabular}

Note. ${ }^{*}$ Correlation is significant $(p<.01)$. NA - negative affectivity; SI - social inhibition.

Zahra Mousavi, Farzaneh Hamidi,

Mohammad

Saadati,

Ali Taghizadieh,

Parastoo Amiri,

Neda Gilani

\section{Figure 2}

Non-mediated $(A)$ and mediated (B) pathways between type D personality and the occurrence of COVID-19. The paths' unstandardized regression coefficients (b) with the standard errors (SE)

(A)

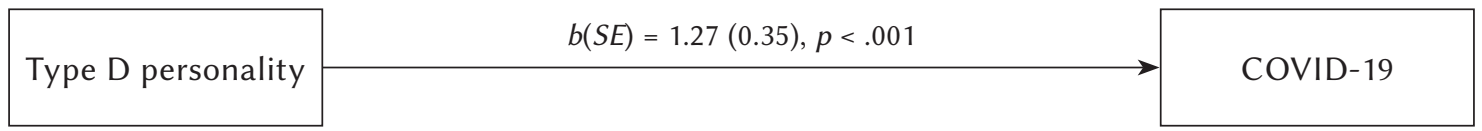

(B)

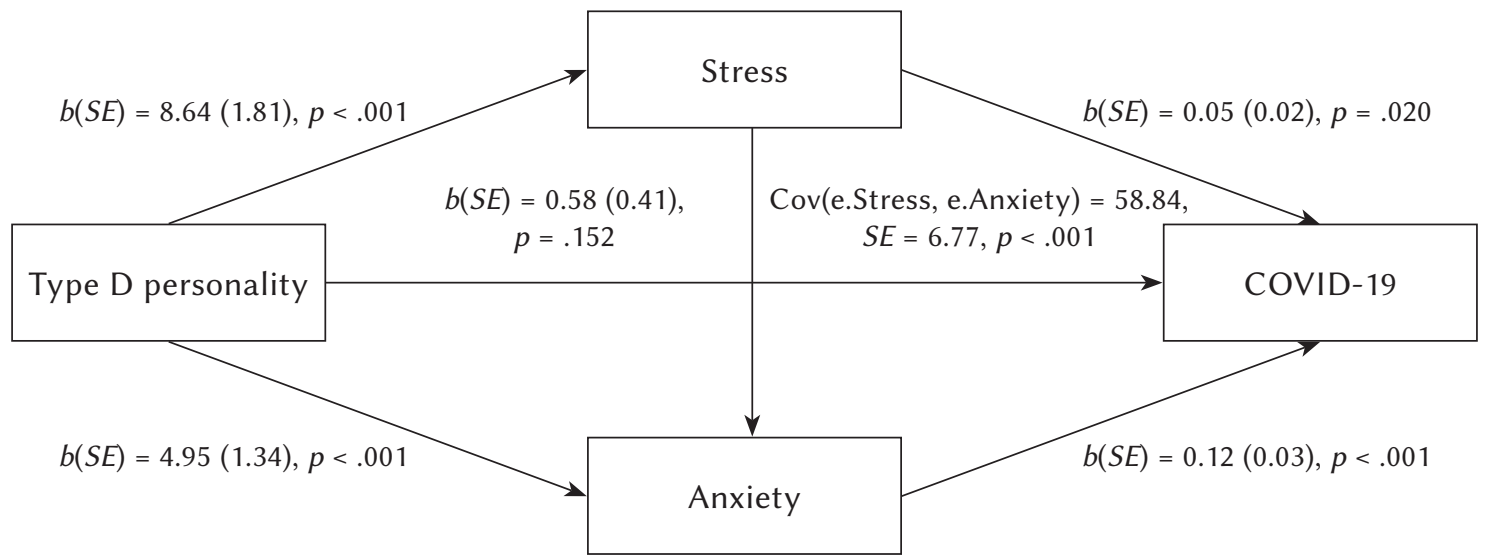

personality is a factor contributing to COVID-19 infection $(p<.001)$. Nonetheless, given that the relationship between type $\mathrm{D}$ personality and infection is not significant in model B $(p=.147)$, the mediating variables of stress and anxiety are recognized as full mediating variables. In addition, there was a direct relationship between anxiety and stress $(p<.001)$.

Table 4 shows the direct effects of type D personality and stress and anxiety on COVID-19 infection in model B, the effect of demographic characteristics and perceived symptoms are included in the model as confounder variables. Accordingly, the type D personality has no significant direct effect on infection with this virus $(p=.147)$. For every unit increase in the stress score, the odds of infection increase by $4 \%$ in those visiting the hospitals $(\mathrm{OR}=1.04$, $p=.045)$, and for every unit increase in the anxiety score, the odds of infection increase in them by $12 \%(\mathrm{OR}=1.12, p<.001)$. Furthermore, taking into account the mediating variable of stress, the odds of infection with COVID-19 in people with type D personality are 2.21 times higher than those without this personality trait $(\mathrm{OR}=2.21, p=.027)$. Moreover, taking into account the mediating variable of anxiety, having a type $\mathrm{D}$ personality increases the odds of infection 2.62-fold ( $\mathrm{OR}=2.62, p=.011$ ), holding demographic characteristics and perceived symptoms constant. 


\section{DISCUSSION}

The present study used statistical modeling in an attempt to determine the role of stress and anxiety as mediators in the relationship with COVID-19 infection, holding demographic characteristics and perceived symptoms constant. According to the results, type D personality can be linked to COVID-19 mediated by stress and anxiety, although it does not have a significant direct relationship with COVID-19. Overall, no significant difference was found between the infected and non-infected groups in terms of age, gender, education, marital status, and BMI, but there were significant differences between them in terms of urban vs. rural residence and occupation. Accordingly, housewives/retired people had the highest percentage of infection and the percentage of infection was significantly higher in the urban residents than the rural residents. Various studies have shown that epidemic diseases such as COVID-19 can be related to demographic characteristics (Haghshenas et al., 2008; Modersitzki et al., 2021; Volk et al., 2020).

In our study, the most common symptoms were vomiting, stomach ache, and fever. Also, we found that fever, cough, vomiting, and stomach ache were associated with a positive test outcome. The findings of this study indicated a positive and significant relationship among all the variables including type D and its subdomains (NA, SI), stress, and anxiety. This finding also agreed with the mediating variable model showing that type D personality, stress and anxiety have an effect on infection with COVID-19. Given that the relationship between type D personality and COVID-19 infection was not significant, the mediating variables of stress and anxiety are identified as full mediating variables. Without taking into account the mediating variables of stress and anxiety, type D personality is a factor contributing to COVID-19 infection. While previous research has also confirmed this link, since studies have shown that people with type D personality are more predisposed to developing underlying diseases (Esmailpour et al., 2013; Volk et al., 2020), few studies have been able to determine the relationships between the components and mediators affecting this pathway.

In the present study, for every unit increase in the stress score, the odds of infection increase by $5 \%$ in those visiting the hospitals, and for every unit increase in the anxiety score, the odds of infection increase by $12 \%$. Similarly, Ramezani et al. (2020) reported higher levels of cortisol and anxiety in patients who later died of COVID-19 than in the survivors. Moreover, taking into account the mediating variable of stress, the odds of infection with COVID-19 in people with type D personality were 3.19 times higher than in those without this personality, and taking into account the mediating variable

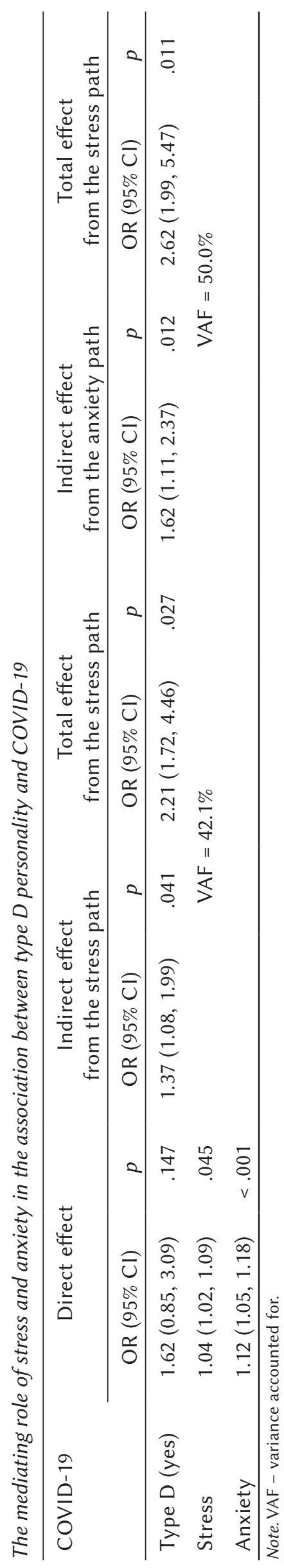

Type D personality and COVID-19 
of anxiety, having a type $\mathrm{D}$ personality increased the odds of infection 2.72-fold. In addition to their high-risk lifestyles and high risk of infection, people with type D personality appear to suffer from chronic stress and anxiety too. Physiologically, stress and anxiety can affect the immune system, thereby weakening the body's defense system (Gilani et al., 2019; Haghshenas et al., 2008; Ray et al., 2017; Speth et al., 2020). As such, these people are probably more exposed to infection with COVID-19. However a previous study showed that the personality dimensions did not mediate the probable risk for posttraumatic stress disorder during the acute COVID-19 outbreak (Ranieri et al., 2021). The statistical analysis, which took account of covariance between anxiety and stress, indicated the direct and significant relationship between anxiety and stress, which is consistent with previous research (Dymecka et al., 2021).

Nonetheless, from another perspective, infection with this virus can have several psychological effects in people with this type of personality. It has been reported that one of the potential long-lasting consequences of COVID-19 is emotional disorder in the patient (Speth et al., 2020). Depression and anxiety had a positive relationship with COVID-19 symptoms such as the loss of smell and taste, but no relationship was observed between mood and depression with COVID-19 symptoms such as fever, cough, or shortness of breath, which may suggest a poor prognosis (Speth et al., 2020). Given that the various aspects of this disease have not yet been fully identified and care must be taken in dealing with these patients, more accurate longitudinal studies in the future can help further reveal the features of this disease.

\section{LIMITATIONS}

Since the present study was conducted during the risky times of the COVID-19 outbreak, it had certain limitations, including its cross-sectional design; also, the people visiting hospitals at this time may have been overcome by the stress and anxiety of possible infection. The number of the non-infected people and the infected patients has therefore been influenced by the conditions of the time and place. Given the study type, it was assumed that the participants completed the questionnaire honestly. Considering that the questionnaires were completed in medical centers, the percentage of infected patients completing the questionnaires may have been higher; therefore, care must be taken in the generalization of the results to the entire society. In addition to anxiety and stress, other mediators of the relationship between the type D personality and COVID-19 might exist, so it is suggested to examine them in future research.

\section{CONCLUSIONS}

Although no relationship was observed between the demographic characteristics of the participants and COVID-19, the geographical place of residence and occupation were found to potentially affect infection with this virus. A positive and significant relationship was observed between all the variables including type D personality, stress, and anxiety, and disregarding the mediating variables of stress and anxiety, having a type $\mathrm{D}$ personality could contribute to COVID-19 infection. Given the indirect relationship between COVID-19 and type D personality, the mediating variables of stress and anxiety can be identified as full mediating variables. However, many other mediators can also play a role in this area, which requires more extensive studies and the control of other potentially relevant variables. It can be debated whether psychological support activities should be increased and clinical and/or virtual psychological counseling centers should be made more active in order to reduce the rate of stress in society, particularly among individuals with type D personality. Furthermore, considering that the pandemic is continuing, it can be suggested to provide psychological support through online therapies.

\section{References}

Aguayo-Carreras, P., Ruiz-Carrascosa, J. C., \& MolinaLeyva, A. (2020). Type D personality is associated with poor quality of life, social performance, and psychological impairment in patients with moderate to severe psoriasis: a cross-sectional study of 130 patients. Indian Journal of Dermatology, Venereology and Leprology, 86, 375-381. https://doi. org/10.4103/ijdvl.IJDVL_114_19

Allahverdipour, H., Karimzadeh, Z., Alizadeh, N., Asghari Jafarabadi, M., \& Javadivala, Z. (2021). Psychological well-being and happiness among middle-aged women: a cross-sectional study. Health Care for Women International, 42, 28-42. https://doi. org/10.1080/07399332.2019.1703990

Antony, M. M., Bieling, P. J., Cox, B. J., Enns, M. W., \& Swinson, R. P. (1998). Psychometric properties of the 42-item and 21-item versions of the Depression Anxiety Stress Scales in clinical groups and a community sample. Psychological Assessment, 10, 176-181. https://doi.org/10.1037/1040-3590.10.2.176

Bagherian Sararoudi, R., Afshar, H., Adibi, P., Daghaghzadeh, H., Fallah, J., \& Abotalebian, F. (2011). Type D personality and quality of life in patients with irritable bowel syndrome. Journal of Research in Medical Sciences, 16, 985-992.

Beaujean, A. A., \& Parker, S. (2020). Path analysis in personality research. In B. J. Carducci, C. S. Nave, J. S. Mio, \& R. E. Riggio (Eds.), The Wiley 
encyclopedia of personality and individual differences: Measurement and assessment (pp. 125-130). Wiley.

Borkoles, E., Kaiseler, M., Evans, A., Ski, C. F., Thompson, D. R., \& Polman, R. C. (2018). Type D personality, stress, coping and performance on a novel sport task. PloS One, 13, e0196692. https://doi.org/ 10.1371/journal.pone.0196692

Chen, S., Yang, J., Yang, W., Wang, C., \& Bärnighausen, T. (2020). COVID-19 control in China during mass population movements at New Year. The Lancet, 395, 764-766. https://doi.org/10.1016/ S0140-6736(20)30421-9

Denollet, J. (1998). Personality and coronary heart disease: the type-D scale-16 (DS-16). Annals of Behavioral Medicine, 20, 209-215. https://doi.org/10.1007/ BF02884962

Denollet, J. (2000). Type D personality: a potential risk factor refined. Journal of Psychosomatic Research, 49, 255-266. https://doi.org/10.1016/s00223999(00)00177-x

Denollet, J. (2005). DS-14: standard assessment of negative affectivity, social inhibition, and type D personality. Psychosomatic Medicine, 67, 89-97. https://doi.org/10.1097/01.psy.0000149256.81953.49

Denollet, J., \& Pedersen, S. (2009). Anger, depression, and anxiety in cardiac patients: the complexity of individual differences in psychological risk. Journal of the American College of Cardiology, 53, 947-949. https://doi.org/10.1016/j.jacc.2008.12.006

Denollet, J., Sys, S. U., \& Brutsaert, D. L. (1995). Personality and mortality after myocardial infarction. Psychosomatic Medicine, 57, 582-591. https:// doi.org/10.1097/00006842-199511000-00011

Dymecka, J. (2021). Psychosocial effects of the COVID-19 pandemic. Neuropsychiatry and Neuropsychology, 16, 1-10. https://doi.org/10.5114/nan. 2021.108030

Dymecka, J., Gerymski, R., \& Machnik-Czerwik, A. (2021). Fear of COVID-19 as a buffer in the relationship between perceived stress and life satisfaction in the Polish population at the beginning of the global pandemic. Health Psychology Report, 9, 149-159. https://doi.org/10.5114/hpr.2020.102136

Esmailpour, K., Babapour, J., \& Ebrahimiesarindizaj, Z. (2013). The relationship between HEXACO personality dimensions and type D personality. Journal of Research and Health, 3, 405-411.

Gilani, N., Haghshenas, R., \& Esmaeili, M. (2019). Application of multivariate longitudinal models in SIRT6, FBS, and BMI analysis of the elderly. The Aging Male, 22, 260-265. https://doi.org/10.1080/ 13685538.2018.1477933

Gilmour, J., \& Williams, L. (2012). Type D personality is associated with maladaptive health-related behaviours. Journal of Health Psychology, 17, 471-478. https://doi.org/10.1177/1359105311423117

Haghshenas, R., Marandi, S. M., Molavi, H., \& Khayyambashi, K. (2008). Predicting injuries of athletes by considering psychological factors. World Journal of Sport Science, 1, 38-41.

Hair, J. F., Sarstedt, M., Ringle, C. M., \& Gudergan, S. P. (2017). Advanced issues in partial least squares structural equation modeling. Sage Publications.

Henry, J. D., \& Crawford, J. R. (2005). The short-form version of the Depression Anxiety Stress Scales (DASS-21): Construct validity and normative data in a large non-clinical sample. British Journal of Clinical Psychology, 44, 227-239. https://doi.org/ 10.1348/014466505X29657

Jastrowski Mano, K. E., O’Bryan, E. M., Gibler, R. C., \& Beckmann, E. (2019). The co-occurrence of pediatric chronic pain and anxiety: a theoretical review of a developmentally informed shared vulnerability model. The Clinical Journal of Pain, 35, 989-1002. https://doi.org/10.1097/AJP.0000000000000763

Javadivala, Z., Allahverdipour, H., Asghari Jafarabadi, M., \& Emami, A. (2020). An interventional strategy of physical activity promotion for reduction of menopause symptoms. Health Promotion Perspectives, 10, 383-392. https://doi.org/10.34172/ hpp.2020.57

Kim, Y. H., Kim, S. R., Kim, Y. O., Kim, J. Y., Kim, H. K., \& Kim, H. Y. (2017). Influence of type D personality on job stress and job satisfaction in clinical nurses: The mediating effects of compassion fatigue, burnout, and compassion satisfaction. Journal of Advanced Nursing, 73, 905-916. https://doi. org/10.1111/jan. 13177

Kupper, N., \& Denollet, J. (2007). Type D personality as a prognostic factor in heart disease: Assessment and mediating mechanisms. Journal of Personality Assessment, 89, 265-276. https://doi.org/ 10.1080/00223890701629797

Lovibond, P. F., \& Lovibond, S. H. (1995). The structure of negative emotional states: Comparison of the Depression Anxiety Stress Scales (DASS) with the Beck Depression and Anxiety Inventories. Behaviour Research and Therapy, 33, 335-343. https:// doi.org/10.1016/0005-7967(94)00075-U

Masafi, S., Saadat, S. H., Tehranchi, K., Olya, R., Heidari, M., Malihialzackerini, S., Jafari, M., \& Rajabi, E. (2018). Effect of stress, depression and type D personality on immune system in the incidence of coronary artery disease. Macedonian Journal of Medical Sciences, 6, 1533-1544. https:// doi.org/10.3889/oamjms.2018.217

Modersitzki, N., Phan, L. V., Kuper, N., \& Rauthmann, J. F. (2021). Who is impacted? Personality predicts individual differences in psychological consequences of the COVID-19 pandemic in Germany. Social Psychological and Personality Science, 12, 1110-1130. https://doi.org/10.1177/ 1948550620952576

Mols, F., \& Denollet, J. (2010). Type D personality in the general population: a systematic review of health status, mechanisms of disease, and work-
Type D personality and COVID-19 
related problems. Health and Quality of Life Outcomes, 8, 9. https://doi.org/10.1186/1477-7525-8-9

Mols, F., Thong, M. S., van de Poll-Franse, L. V., Roukema, J. A., \& Denollet, J. (2012). Type D (distressed) personality is associated with poor quality of life and mental health among 3080 cancer survivors. Journal of Affective Disorders, 136, 26-34. https:// doi.org/10.1016/j.jad.2011.08.034

Ramezani, M., Simani, L., Karimialavijeh, E., Rezaei, O., Hajiesmaeili, M., \& Pakdaman, H. (2020). The role of anxiety and cortisol in outcomes of patients with COVID-19. Basic and Clinical Neuroscience, 11, 179-184. https://doi.org/10.32598/bcn.11. covid19.1168.2

Shahsavarinia, Zeinab Javadivala,

Hamid

Allahverdipour,

Zahra Mousavi,

Farzaneh Hamidi,

Mohammad

Saadati,

Ali Taghizadieh,

Parastoo Amiri, Neda Gilani

Ranieri, J., Guerra, F., \& Di Giacomo, D. (2021). Predictive risk factors for post-traumatic stress symptoms among nurses during the Italian acute COVID-19 outbreak. Health Psychology Report, 9, 180-185. https://doi.org/10.5114/hpr.2020.101249

Ray, A., Gulati, K., \& Rai, N. (2017). Stress, anxiety, and immunomodulation: a pharmacological analysis. Vitamins and Hormones, 103, 1-25. https:// doi.org/10.1016/bs.vh.2016.09.007

Raykh, O. I., Sumin, A. N., \& Korok, E. V. (2021). The influence of personality type D on cardiovascular prognosis in patients after coronary artery bypass grafting: Data from a 5-year-follow-up study. International Journal of Behavioral Medicine. https:// doi.org/10.1007/s12529-021-09992-y

Reed, R. G., \& Raison, C. L. (2016). Stress and the immune system. In C. Esser (Ed.), Environmental influences on the immune system (pp. 97-126). Springer.

Samani, S., \& Joukar, B. (2007). A study on the reliability and validity of the short form of the Depression, Anxiety, Stress Scale (DASS-21). Journal of Social Sciences and Humanities of Shiraz University, 26, 65-77.

Schou-Bredal, I., Grimholt, T., Bonsaksen, T., Skogstad, L., Heir, T., \& Ekeberg, Ø. (2021). Optimists' and pessimists' self-reported mental and global health during the COVID-19 pandemic in Norway. Health Psychology Report, 9, 160-168. https:// doi.org/10.5114/hpr.2021.102394

Skalski, S., Uram, P., Dobrakowski, P., \& Kwiatkowska, A. (2020). Thinking too much about the novel coronavirus. The link between persistent thinking about COVID-19, SARS-CoV-2 anxiety and trauma effects. Current Issues in Personality Psychology, 8, 169-174. https://doi.org/10.5114/cipp.2020.100094

Speth, M. M., Singer-Cornelius, T., Oberle, M., Gengler, I., Brockmeier, S. J., \& Sedaghat, A. R. (2020). Mood, anxiety and olfactory dysfunction in COVID-19: Evidence of central nervous system involvement? The Laryngoscope, 130, 2520-2525. https://doi.org/10.1002/lary.28964

Tuman, T. C. (2021). The effect of type D personality on anxiety, depression and fear of COVID-19 disease in healthcare workers. Archives of Environ- mental \& Occupational Health. https://doi.org/10.1 080/19338244.2021.1952152

Volk, A. A., Brazil, K. J., Franklin-Luther, P., Dane, A. V., $\&$ Vaillancourt, T. (2020). The influence of demographics and personality on COVID-19 coping in young adults. Personality and Individual Differences, 168, 110398. https://doi.org/10.1016/j.paid. 2020.110398

Wang, C., Pan, R., Wan, X., Tan, Y., Xu, L., Ho, C. S., \& Ho, R. C. (2020). Immediate psychological responses and associated factors during the initial stage of the 2019 coronavirus disease (COVID-19) epidemic among the general population in China. International Journal of Environmental Research and Public Health, 17, 1729. https://doi.org/10.3390/ ijerph17051729

Xiao, H., Zhang, Y., Kong, D., Li, S., \& Yang, N. (2020). Social capital and sleep quality in individuals who self-isolated for 14 days during the coronavirus disease 2019 (COVID-19) outbreak in January 2020 in China. Medical Science Monitor, 26, e923921. https://doi.org/10.12659/MSM.923921

You, J., Wang, C., Rodriguez, L., Wang, X., \& Lu, Q. (2018). Personality, coping strategies and emotional adjustment among Chinese cancer patients of different ages. European Journal of Cancer Care, 27, e12781. https://doi.org/10.1111/ecc. 12781

Zoghipaidar, M. R., Azish, M., Mazdeh, M., Rezaei, A. A., \& Torabi, A. (2020). Prediction of chronic muscular pain based on personality type, hardiness and perceived social support. Sadra Medical Journal, 8, 321-334. https://doi.org/10.30476/ smsj.2020.82956.1043 